Article

\title{
Simplifying the Preparation of Pollen Grains for MALDI-TOF MS Classification
}

\author{
Franziska Lauer ${ }^{1,2}$, Stephan Seifert ${ }^{1,2,+}$, Janina Kneipp ${ }^{1,2}$ and Steffen M. Weidner ${ }^{2, *}$ \\ 1 Department of Chemistry, Humboldt-University to Berlin, Brook-Taylor-Str. 2, 12489 Berlin, Germany; \\ franziska.lauer@bam.de (F.L.); stephan@familie-seifert.org (S.S.); janina.kneipp@chemie.hu-berlin.de (J.K.) \\ 2 Federal Institute for Materials Research and Testing (BAM), Richard-Willstätter-Str. 11, \\ 12489 Berlin, Germany \\ * Correspondence: steffen.weidner@bam.de; Tel.: +49-30-8104-1633 \\ + Present Address: Institute of Medical Informatics and Statistics, Kiel University, Brunswiker Str. 10, \\ 24105 Kiel, Germany
}

Academic Editors: David Arráez-Román and Ana Maria Gómez-Caravaca

Received: 31 January 2017; Accepted: 27 February 2017; Published: 3 March 2017

\begin{abstract}
Matrix-assisted laser desorption ionization time of flight mass spectrometry (MALDI-TOF MS) is a well-implemented analytical technique for the investigation of complex biological samples. In MS, the sample preparation strategy is decisive for the success of the measurements. Here, sample preparation processes and target materials for the investigation of different pollen grains are compared. A reduced and optimized sample preparation process prior to MALDI-TOF measurement is presented using conductive carbon tape as target. The application of conductive tape yields in enhanced absolute signal intensities and mass spectral pattern information, which leads to a clear separation in subsequent pattern analysis. The results will be used to improve the taxonomic differentiation and identification, and might be useful for the development of a simple routine method to identify pollen based on mass spectrometry.
\end{abstract}

Keywords: MALDI-TOF MS; conductive carbon tape; pollen; sample pretreatment; principal component analysis

\section{Introduction}

Matrix-assisted laser desorption ionization mass spectrometry (MALDI MS) has become a suitable technique to explore complex biological materials, such as small organisms (e.g., ticks, flies, mites etc.) [1-3], microorganisms [4-6], or biological particles (e.g., pollen grains) [7]. For this purpose, various sample preparation procedures have been modified, which involved grinding of organisms [8], suspension/extraction methods [3,6] or the dissection of organs [2].

Besides the digestion/extraction step, the fixation of such samples represents a crucial step for the success of the experiment. In contrast to MALDI MS of samples that are deposited on the target plate by being embedded in a matrix, or by covering tissue sections obtained from microtomes with a matrix [9-11], these samples have to be fixed by alternative methods. Widely known is the application of surface-enhanced laser desorption ionization time-of-flight mass spectrometry (SELDI-TOF MS) [12]. There, the target holders incorporate either modified chemical spots (hydrophobic, cationic, anionic, metal ions or hydrophilic), suited for protein expression profiling studies, or pre-activated biological surfaces designed for coupling of biomolecules [12]. A recent overview of the application of SELDI-TOF MS for the discovery of biomarkers of various forms of human cancer is given by Gopal et al. [13]. However, this technique is limited to the investigation of analytes with comparatively low molecular masses. 
Another approach is the fixation of samples in adhesives. Kaftan et al. used epoxy glue for the fixation of flies [1]. Recently, we showed that pollen grains could be fixed in a resin typically used for atomic force microscopy (AFM) applications, without affecting the extraction of analytes or the ionization procedure in MALDI-TOF MS [14]. In this approach, we showed recently that pollen grains could be fixed without affecting the extraction of analytes or the ionization procedure in MALDI-TOF MS. This approach, however, is time-consuming, since it involves various steps. First, pollen grains are deposited on a resin film that has to be prepared on the target before. Afterwards, the target plate is exposed to solvent vapor that weakens the resin and enables the pollen grains to be fixed in the viscous film. In a subsequent step, the fixed pollen samples are exposed to formic acid atmosphere, with the purpose of extracting the analytes from the pollen grain. Finally, the layer is covered by a matrix. It becomes obvious that this approach is not applicable for fast and automated pollen monitoring. Thus, we focused our efforts on the development of alternative methods for fast pollen grain fixation for MALDI MS probing.

For pollen classification and identification, the applicability of MALDI-TOF MS was proven, combining the mass spectral data with hierarchical cluster analysis (HCA) and principal component analysis (PCA) [15]. There, almost 200 pollen samples of 74 different species from 11 genera and 2 plant orders were classified according to their taxonomic relationships, including discrimination of species that feature a very high chemical similarity [15]. The application of multivariate statistics (e.g., PCA) to mass spectral patterns yields an enormous improvement concerning taxonomic classification of the samples compared to common microscopic techniques.

In order to simplify the sample preparation process, we suggest the application of a sticky conductive carbon tape. Conductive tape was used in this context previously for the analysis of methamphetamine incorporated in hair [16]. A recent application was reported by Kajiwara et al., where spider mites were fixed on a double-sided carbon tape and analyzed by MALDI-TOF MS [8]. In this article, we demonstrate that the use of conductive carbon tape on a MALDI target facilitates the pollen sample treatment, compared to a conventional sample deposition on a steel target. Different preparation strategies are compared, e.g., regarding the influence of acid concentration in the matrix solution and additional extraction with liquid or gaseous acid. In addition, possible interferences of the formic acid used for extraction with the new target material are examined. The findings show that our approach enhances the information that can be taken from the species-specific mass peak patterns. This results in a clear differentiation in subsequent pattern analysis, which is important when analyzing pollen grains in various real mixtures.

\section{Results}

Our experiment included pollen grains from four tree species: Scots pine (Pinus sylvestris), Japanese bog birch (Betula tatewakiana), Italian aider (Alnus cordata) and common hazel (Corylus avellana). The first sample represents the plant family of Pinaceae in the order of Coniferales, the other samples belong to the plant family of Betulaceae in the order of Fagales.

Spectra from all samples were recorded on conductive tape and on a stainless steel target, respectively. In Figure 1, the average spectra from five repetitive measurements obtained with different sample treatments and target materials are depicted exemplarily for Alnus cordata. All average spectra showed similar peak patterns, suggesting that these peak patterns are characteristic of this Alnus cordata sample. However, slight shifts in the accurate masses and differences in signal intensities can be detected using different sample treatments. Spectra, which were recorded on the conductive tape (Figure 1a-f) generally showed higher absolute signal intensities compared to those with the same treatment strategies measured on the steel target (Figure 1g-1). Adding trifluoroacetic acid (TFA) to the matrix solution (Figure 1a-c,g-i) also resulted in increased signal intensities, compared to the respective spectra obtained without TFA addition (Figure $1 \mathrm{~d}-\mathrm{f}, \mathrm{j}-\mathrm{l}$ ). When the extraction procedure was conducted with an additional droplet deposition of formic acid onto the pollen grains (Figure 1c,f,i,l), spectra were obtained that feature low signal intensities, even though the individual peaks were 
clearly distinguishable. In the spectra obtained after formic acid gas phase extraction of the sample (Figure 1b,e,h,k), the background in the lower mass range as well as the absolute intensities were more intense than in the spectra gained from droplet extraction. Spectra of the pollen samples with no additional extraction step (Figure $1 \mathrm{a}, \mathrm{d}, \mathrm{g}, \mathrm{j}$ ) provided signal intensities in the lower mass range that are equal to those of the spectra obtained with gas phase extraction, whereas higher mass peaks (e.g., at $m / z 6891$ and $m / z$ 9489) were more intense. The most intense spectrum showing characteristic masses of Alnus cordata was recorded on conductive tape using a mixture of $\mathrm{ACN} / \mathrm{H}_{2} \mathrm{O}(1.25 \%$ TFA) as the matrix solvent and without any additional extraction steps (Figure 1a).

The corresponding mass spectra of the other pollen species (Betula tatewakiana, Corylus avellana and Pinus sylvestris) are shown in the Appendix A (Figures A1-A3). As indicated by the intensities of the corresponding signals, the use of a matrix solvent containing TFA promoted the extraction of analytes from the exines, i.e., the pollen grains' outer shells, of most of these pollen samples much more than an additional extraction step using formic acid (for example compare Figure A2a,h with Figure A2c,f,i,l; or Figure A3a-c with Figure A3b,e,h,k). With the exception of the birch pollen investigations (Betula tatewakiana) (Figure A1), our previous findings concerning Alnus cordata spectra in Figure 1 were confirmed by the analysis of additional pollen species.

The study of the birch pollen sample with formic acid droplet deposition on the MTP (microtiter plate) steel target holder (Figure A1i,l) yielded intense spectra that showed a very low background in the lower mass range. In that case, the use of conductive tape in combination with a matrix solvent containing TFA and no additional extraction steps (Figure A1a) did not provide the spectra with the highest quality. Nevertheless, the spectrum also showed the characteristic peak pattern (as in Figure A1i,l) that could be analyzed by multivariate tools, as will be discussed below.

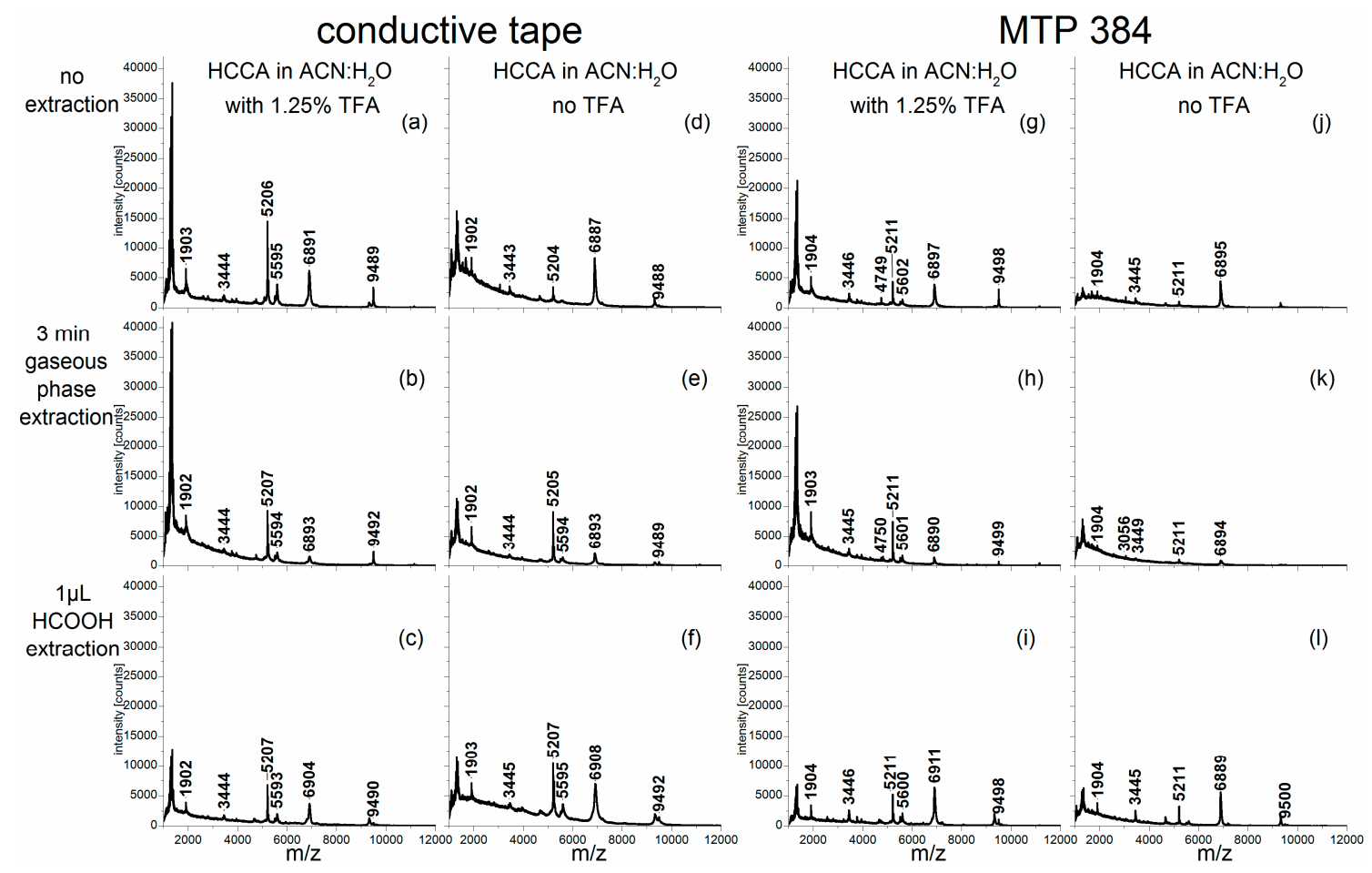

Figure 1. Averaged matrix-assisted laser desorption ionization time of flight (MALDI-TOF) mass spectra $(n=5)$ of Alnus cordata pollen grains: measured on conductive tape with trifluoroacetic acid (TFA) in the matrix solution $(\mathbf{a}-\mathbf{c})$, on conductive tape without TFA in the matrix solution ( $\mathbf{d}-\mathbf{f})$, on the steel target (microtiter plate MTP 384) with TFA in the matrix solution (g-i) and on the steel target without TFA in the matrix solution ( $\mathbf{j}-1)$ (in the rows: extraction procedure). 
At first glance, the spectra of all four pollen species show species-specific peak patterns, which already makes a rough differentiation of species possible by comparing the spectra by eye (Figures 1 and A1, Figures A2 and A3). In addition to this, an objective differentiation that enables an evaluation of particular sample treatment methods for the purpose of classification and ultimately identification can be obtained by using multivariate data analysis.

Multivariate methods, such as principal component analysis (PCA) can be applied to mass spectrometric data for several reasons, e.g., for quality control, to reduce complexity of the data, and to emphasize differences within a data set that enable classification [17-20].

We applied PCA to compare classification of pollen species upon the application of different preparation procedures and to evaluate the preparation setup by finding differences in the chemical composition of the samples. Principal components (PCs) are linear combinations of the original mass spectra and generate a new coordinate system that is based on maximum variance between the spectra [21]. They are characterized by two matrices: the loadings and the scores, as well as the percentage of original variance they are representing [22-24]. The scores of the first and second principal components of the PCA using the respective spectra (Figure 1, Figures A1-A3) after pre-treatment (see Section 4.4 for further information) are depicted in Figure 2. In order to compare the separation of the pollen species, the scores plot of each PCA was evaluated regarding the intra- versus inter-species distances. The loadings, given in Figure 3, show the connection between the principal components and the original data and can be used for the subsequent interpretation of the scores values (Figure 2). They indicate which parts of the spectrum are relevant for the respective PC and lead to the separation of the pollen spectra in the scores plots of Figure 2.

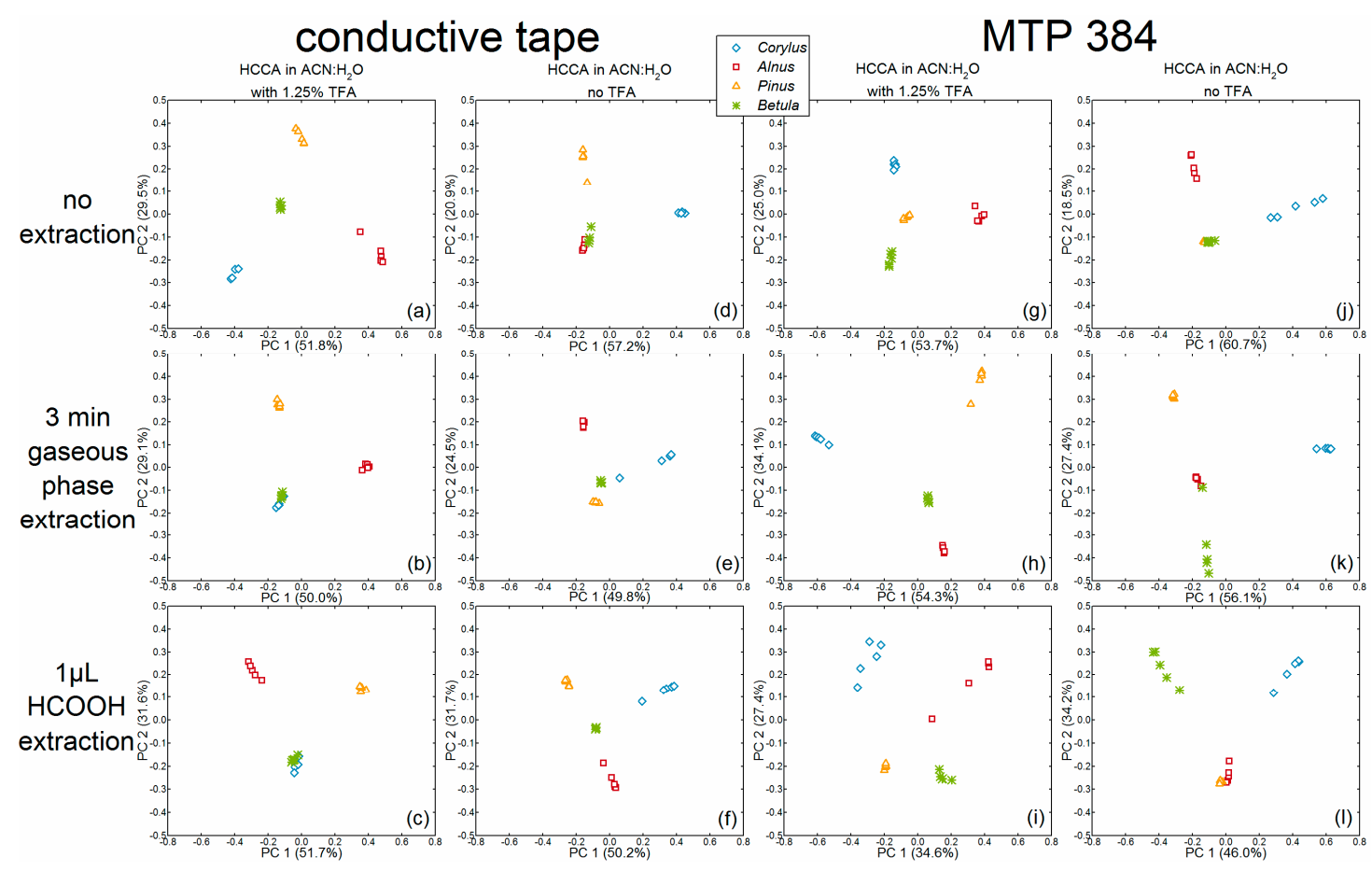

Figure 2. Scores and variances of the first and second principal components (PC) of the PCA (principal component analysis) of MALDI MS spectra from four pollen species (Corylus avellana, Alnus cordata, Pinus sylvestris, Betula tatewakiana) for different sample preparation techniques (rows: variation in extraction procedure ((a,d, $\mathbf{g}, \mathbf{j})$ : without additional extraction); (b,e, $\mathbf{h}, \mathbf{k})$ : gaseous extraction); $(\mathbf{c}, \mathbf{f}, \mathbf{i}, \mathbf{l})$ : droplet extraction)); columns: variation in target material and matrix solution $((\mathbf{a}-\mathbf{c})$ : conductive tape with trifluoroacetic acid in the matrix solution); (d-f): CT without TFA in the matrix solution); (g-i): steel target, matrix with TFA); and (j-1): steel target, matrix without TFA)). Data pre-treatment is explained in the methods Section 4.4. 
The first parameter to be discussed using the PCA scores plots is the addition of TFA in the matrix solutions: PCAs conducted on spectra obtained without TFA in the matrix solution (Figure $2 \mathrm{~d}-\mathrm{f}, \mathrm{j}-\mathrm{l}$ ) shared a common trait, that their respective first PC enables a separation of Corylus avellana spectra (light-blue diamonds) by positive values from negative or zero values of the spectra from all other species. In accord with this, the Corylus-typical spectral regions, e.g., from $m / z 5400$ to 6117 [15], yielded positive signals in the loadings of the respective first PCs (see Figure 3, 2nd and 4th column, black loadings spectra). The other species, however, cannot be differentiated clearly from each other using the scores plots in Figure 2d,e,j-l. The scores of the second and third PCs of the respective data sets, did not allow clear and accurate differentiation between the species Alnus cordata, Pinus sylvestris and Betula tatewakiana (see Figure A4) either.
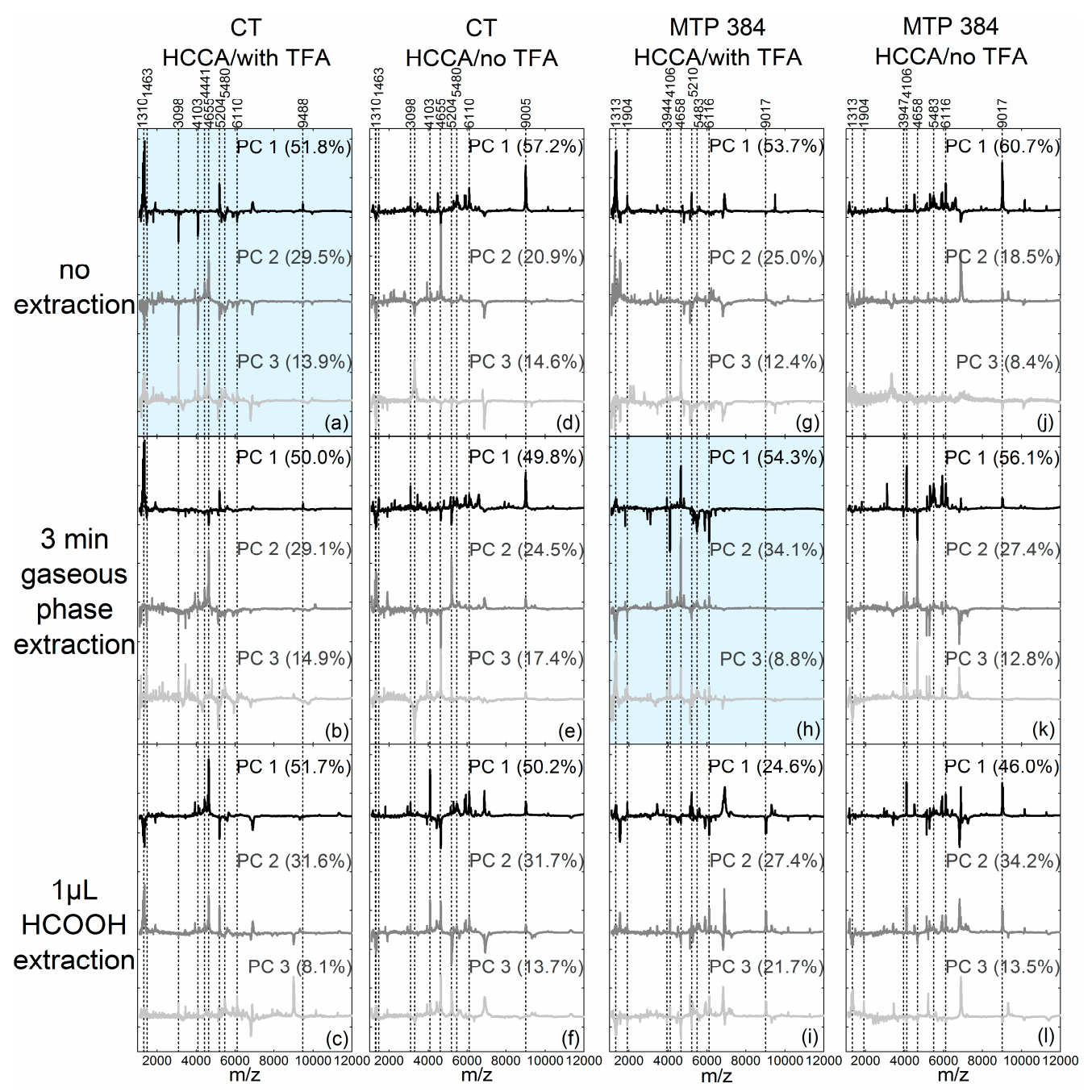

Figure 3. Loadings and variances (in brackets) of the first PC (top, black), the second PC (middle, gray) and the third PC (bottom, light gray) of the respective PCAs (rows: variation in extraction procedure $((\mathbf{a}, \mathbf{d}, \mathbf{g}, \mathbf{j})$ : without additional extraction); $(\mathbf{b}, \mathbf{e}, \mathbf{h}, \mathbf{k})$ : gaseous extraction); $(\mathbf{c}, \mathbf{f}, \mathbf{i}, \mathbf{l})$ : droplet extraction)); columns: variation in target material and matrix solution $((\mathbf{a}-\mathbf{c})$ : conductive tape with trifluoroacetic acid in the matrix solution); $(\mathbf{d}-\mathbf{f})$ : CT without TFA in the matrix solution); $(\mathrm{g}-\mathbf{i})$ : steel target, matrix with TFA); and (j-1): steel target, matrix without TFA)), for better comparison, graph $(\mathbf{a}, \mathbf{h})$ are highlighted.

In the PCAs of the data sets that were obtained with the addition of TFA in the matrix solution, a separation of individual pollen species using the first two PCs was possible more frequently, as indicated in the plots illustrated in Figure 2a,g,h,i. However, in contrast to the plots in Figure 2a,h, 
where the individual groups were clearly separated from each other, the distances of the different groups in the plot in Figure $2 \mathrm{~g}$ were much smaller. Moreover, considering the homogeneity of each individual group in these four plots, the plot in Figure $2 \mathrm{i}$ especially showed very inhomogeneous distribution of the spectra of Alnus cordata and Corylus avellana. Since high intra-species variances are especially noticeable in the bottom row of Figure $2 c, f, i, l$ they could possibly be explained by the samples treatment with the formic acid droplet method. Different to this observation, the PCAs of data obtained by additional gaseous extraction (Figure $2 \mathrm{~b}, \mathrm{e}, \mathrm{h}, \mathrm{k}$ ) and by no additional extraction step (Figure $2 \mathrm{a}, \mathrm{d}, \mathrm{g}, \mathrm{j}$ ) showed no definite tendency concerning intra-species variances.

Since the scores plots in Figure 2a, h are suitable for a complete differentiation of pollen species, the underlying PCAs of these data shall be discussed in detail here. In Figure 2a, the scores of Corylus avellana spectra feature negative values for the first and second PCs, which result in negative Corylus-typical peaks [15] in the respective loadings (e.g., at $m / z 3098, m / z 4103$ and the range from $m / z 5400$ to 6110 shown in Figure 3a, top black spectra and middle gray spectra). Alnus cordata spectra provide scores values that are positive for the first PC and negative for the second PC (Figure 2a). The corresponding loadings (Figure 3a) showed peaks in the lower mass range of $m / z 1310$ to 1463 and peaks around $m / z 5204$ and $m / z 9488$ that can be assigned to Alnus [15]. The Pinus sylvestris spectra were characterized by scores values close to zero for the first PC and very positive score values for the second PC. Consequently, the loadings of the second PC in Figure 3a showed peaks at $m / z 4441$ and $m / z 4655$ [15] that do not occur in the loadings of the first PC and that can be identified as Pinus-typical (compare with Figure A3a). The Betula tatewakiana scores values in Figure 2a were all close to zero, therefore the loadings showed no peaks that can be assigned to Betula pollen spectra.

In Figure 2h, Corylus avellana spectra yield negative scores values for the first PC and positive scores values for the second PC. Hence, the respective loadings (Figure 3h) showed negative and positive Corylus-typical peaks in the top black and the middle gray loadings, respectively (e.g., at $m / z 4106$ and the mass range from $m / z 5400$ to 6116). Furthermore, the Alnus cordata spectra in Figure $2 \mathrm{~h}$ had zero values for the first PC and negative values for the second PC. Thus, in the loadings of the second PC in Figure 3h, negative values for the Alnus-typical peak pattern in the lower mass range $(m / z 1313$ to 1400 and peaks around $m / z$ 1904) can be found (compare with Figure $1 \mathrm{~h}$ ). The Pinus sylvestris spectra featured positive scores values for the first and second PCs (Figure 2h), and Pinus-typical peaks at $\mathrm{m} / \mathrm{z} 3944$ and $\mathrm{m} / \mathrm{z} 4658$ can be observed in the corresponding loadings in Figure $3 \mathrm{~h}$, top black and middle gray loadings). Betula tatewakiana spectra in the PCA of Figure $2 \mathrm{~h}$, again, were characterized by scores values around zero, which result in loadings that did not show Betula-typical characteristics.

\section{Discussion}

In order to compare different preparation techniques for MALDI-TOF MS classification of pollen, we systematically varied the extraction procedure, the target material and the matrix solution. Our results demonstrated that higher absolute signal intensities could be recorded in the spectra obtained on conductive tape compared to those that were received with the same treatment strategies on the steel target (compare a-f of Figure 1, Figures A1-A3 with g-1 of the corresponding figure). Nevertheless, conducting PCA on data sets measured on a steel target was still expedient (Figure 2g-1). Kajiwara et al. [8] observed a slightly higher mass shift and a missing of some lower mass range peaks when mass spectra are obtained on conductive tape. These findings could not be confirmed by our experiments, where the usage of conductive tape as a sample holder facilitated the collection of mass spectra with intense peaks and less noise (a-f of Figure 1, Figures A1-A3). We assume that the use of the sticky conductive tape could be favorable in the extraction by keeping the extracted analytes closer to the pollen grains, and therefore, enabling higher analyte concentrations that lead to more intense signals. The increased sensitivity could be especially important for the detection of single pollen grains in future applications. As shown in a previous investigation based on the MTP approach, MALDI MS is capable of detecting a few, down to $\sim 3$ pollen grains at best [7].

The treatment with acid seems to be indispensable for a sufficient extraction of analytes from the pollen grains (Figure 1, Figures A1-A3). When no acid (formic acid or TFA, see d,j of Figure 1, 
Figures A1-A3) is applied, the MALDI mass spectra exhibited less intense peaks and the different pollen species cannot be differentiated clearly in the PCA (Figure $2 \mathrm{~d}, \mathrm{j}$ ). When a matrix solution without TFA was used, the PCA is dominated by the separation of Corylus avellana spectra from the spectra of the other species, most likely due to less noise and lower background in the Corylus avellana spectra. The other pollen species did not yield spectra of sufficient quality (see d-f,i-l of Figure 1, Figures A1 and A3), leading to higher inner-class variances in the PCA. The addition of $1.25 \%$ TFA to the matrix solution led to an improvement in signal intensity and hence to higher quality spectra (a-c,g-i of Figure 1, Figures A1-A3). As a consequence, the PCA of the data sets that were obtained with a matrix solution containing TFA most often enabled species-specific classification. The formic acid gas phase treatment resulted in intense signals from the birch pollen (Figure A1b), reasonable intensities for Pinus and Alnus pollen (Figures A3b and 1b) and insufficient data for Corylus pollen (Figure A2b). Nevertheless, the PCAs of this dataset showed a clear intra-species homogeneity, even though the scores values of Corylus and Betula-spectra were almost alike. The extraction procedure conducted with formic acid droplet deposition obtained spectra with various intensities for each respective species resulting in large intra-group variance in the PCA. This might be traced back to the non-reproducible evaporation of the formic acid, caused by different droplet spot sizes.

\section{Materials and Methods}

\subsection{Materials}

In this study four different fresh pollen samples (Pinus sylvestris, Betula tatewakiana, Alnus cordata, Corylus avellana) of two plant orders (Coniferales and Fagales), collected from trees in the Botanic Garden of Berlin, were analyzed. After sample collection, the pollen grains were stored at $-20^{\circ} \mathrm{C}$ until usage.

\subsection{Sample Preparation}

For sample preparation, the pollen grains were deposited either separately on an MTP 384 standard target covered with double faced adhesive carbon tape (P77817, Science Services GmbH, Munich, Germany) or directly on a steel target. The following sample treatment procedures were compared using each target.

1. One microliter of HCCA matrix (10 mg of $\alpha$-cyano-4-hydroxycinnamic acid diluted in $1 \mathrm{~mL} \mathrm{1:1}$ acetonitrile/water $(v / v)$ and $1.25 \%$ trifluoroacetic acid) was spotted onto the pollen grains.

2. Pollen grains were deposited on the target, which was kept for $3 \mathrm{~min}$ in a glass box over $98 \%$ formic acid (gas phase extraction). Afterwards, $1 \mu \mathrm{L}$ of the matrix solution (see 1) was deposited.

3. One microliter of formic acid $(98 \%)$ was pipetted onto the pollen grains. After drying at room temperature, $1 \mu \mathrm{L}$ of the matrix solution (see 1 ) was added.

Additionally, these three procedures were repeated using a matrix solution without TFA.

After solvent evaporation, the target containing 48 spots was inserted into the mass spectrometer.

\subsection{Data Acquisition}

An Autoflex III MALDI-TOF mass spectrometer (Bruker Daltonik GmbH, Bremen, Germany) equipped with a $355 \mathrm{~nm}$ Smartbeam laser was used. Spectra were recorded in positive linear mode. Two thousand laser shots were accumulated for one spectrum. The laser settings were kept constant for all spots. From each spot five spectra were recorded at different positions. The instrument was calibrated using biopolymer standards (insulin, cytochrome C, myoglobin, and ubiquitin).

\subsection{Data Analysis}

The spectra pretreatment and multivariate analysis were performed using Matlab software (version R2015a, the Mathworks, Inc., Natick, MA, USA). Raw spectra were interpolated (reduction from 26,834 to 3634 data points) in the mass region between $m / z 1100$ and 12,000 with a step size of 3, baseline-corrected and vector-normalized. Subsequently, principal component analysis (PCA) of the samples that were obtained by the same pretreatment method was performed using the mass range between $\mathrm{m} / z 1100$ and 12,000 . 


\section{Conclusions}

The following two preparation techniques were identified as especially suitable, since intense spectra were gained that featured characteristic mass peaks enabling a distinct classification in the PCA. In sample preparation (a in Figure 1, Figures A1-A3) conductive tape was used as target, a mixture of $\mathrm{ACN} / \mathrm{H}_{2} \mathrm{O}(1.25 \% \mathrm{TFA})$ served as matrix solvent, and no further extraction steps were conducted. The sample preparation (h in Figure 1, Figures A1-A3) was pursued directly on the steel target, with gaseous formic acid extraction and TFA in the matrix solution. However, preparation (a in Figure 1, Figures A1-A3) is by far the simplest sample preparation procedure; the pollen grains can be fixed easily on the conductive tape, and no further extraction steps must follow. Therefore, we suggest this procedure to establish a preoperatively simple and reliable routine method for pollen analysis based on MALDI-TOF MS. Future work will focus on the improvement of the sensitivity of this technique and the combination of MALDI imaging with multivariate data analysis.

Acknowledgments: The research group thanks Thomas Dürbye of the Botanic Garden and Botanical Museum Berlin-Dahlem for the support in sample collection. Janina Kneipp acknowledges funding by European Research Council (ERC) grant no. 259432.

Author Contributions: Franziska Lauer and Steffen M. Weidner conceived and designed the experiments; Franziska Lauer performed the experiments; Franziska Lauer, Stephan Seifert, Janina Kneipp, and Steffen M. Weidner analyzed the data; Stephan Seifert and Janina Kneipp contributed analysis tools; Franziska Lauer, Stephan Seifert, Janina Kneipp, and Steffen M. Weidner wrote the paper.

Conflicts of Interest: The authors declare no conflict of interest.

\section{Appendix A}

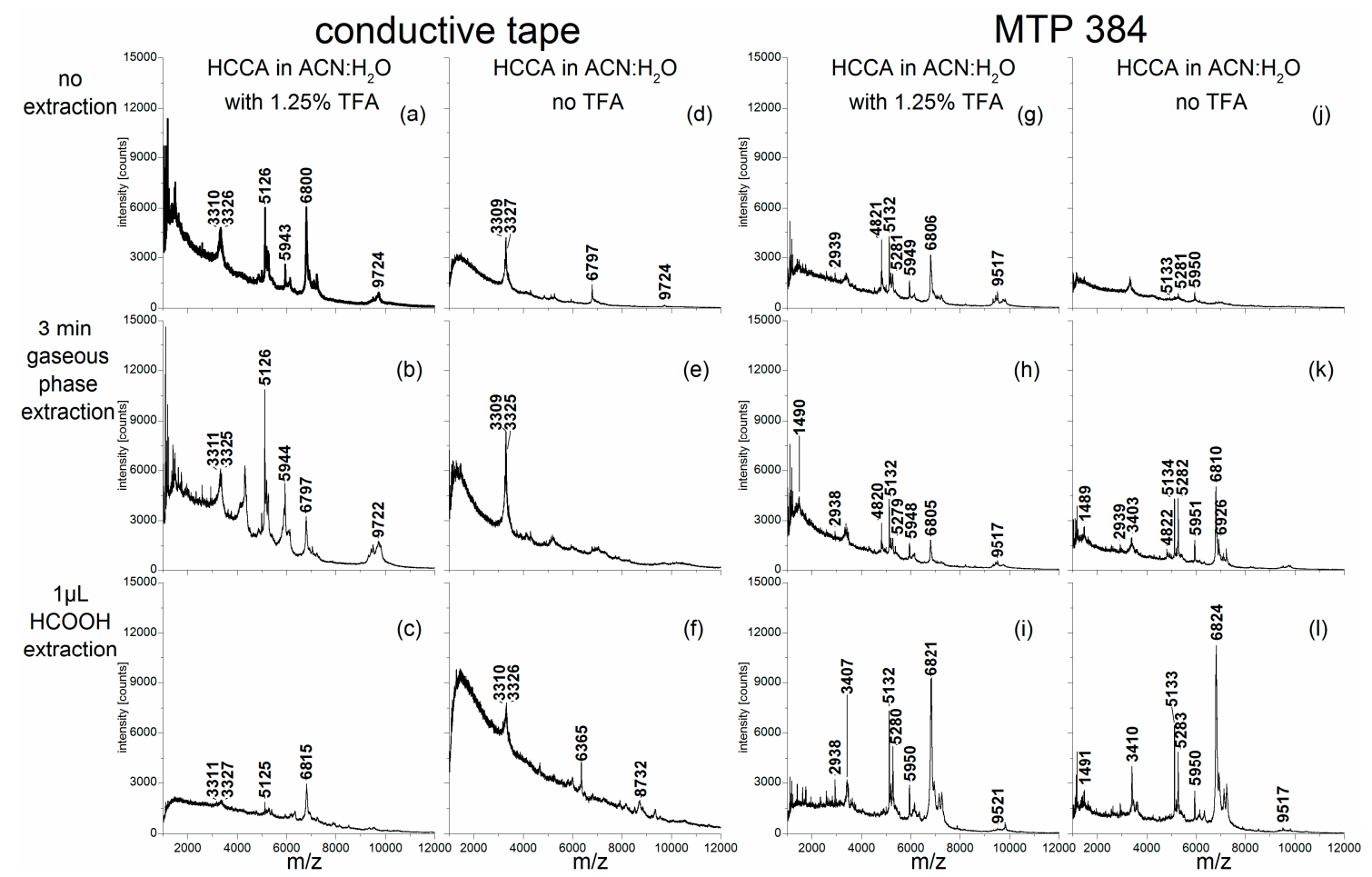

Figure A1. Averaged MALDI-TOF mass spectra $(n=5)$ of Betula tatewakiana pollen grains: measured on conductive tape with TFA in the matrix solution $(\mathbf{a}-\mathbf{c})$, on conductive tape without TFA in the matrix solution (d-f), on the steel target (MTP 384) with TFA in the matrix solution (g-i) and on the steel target without TFA in the matrix solution ( $\mathbf{j}-\mathbf{1})$ (in the rows: extraction procedure). 


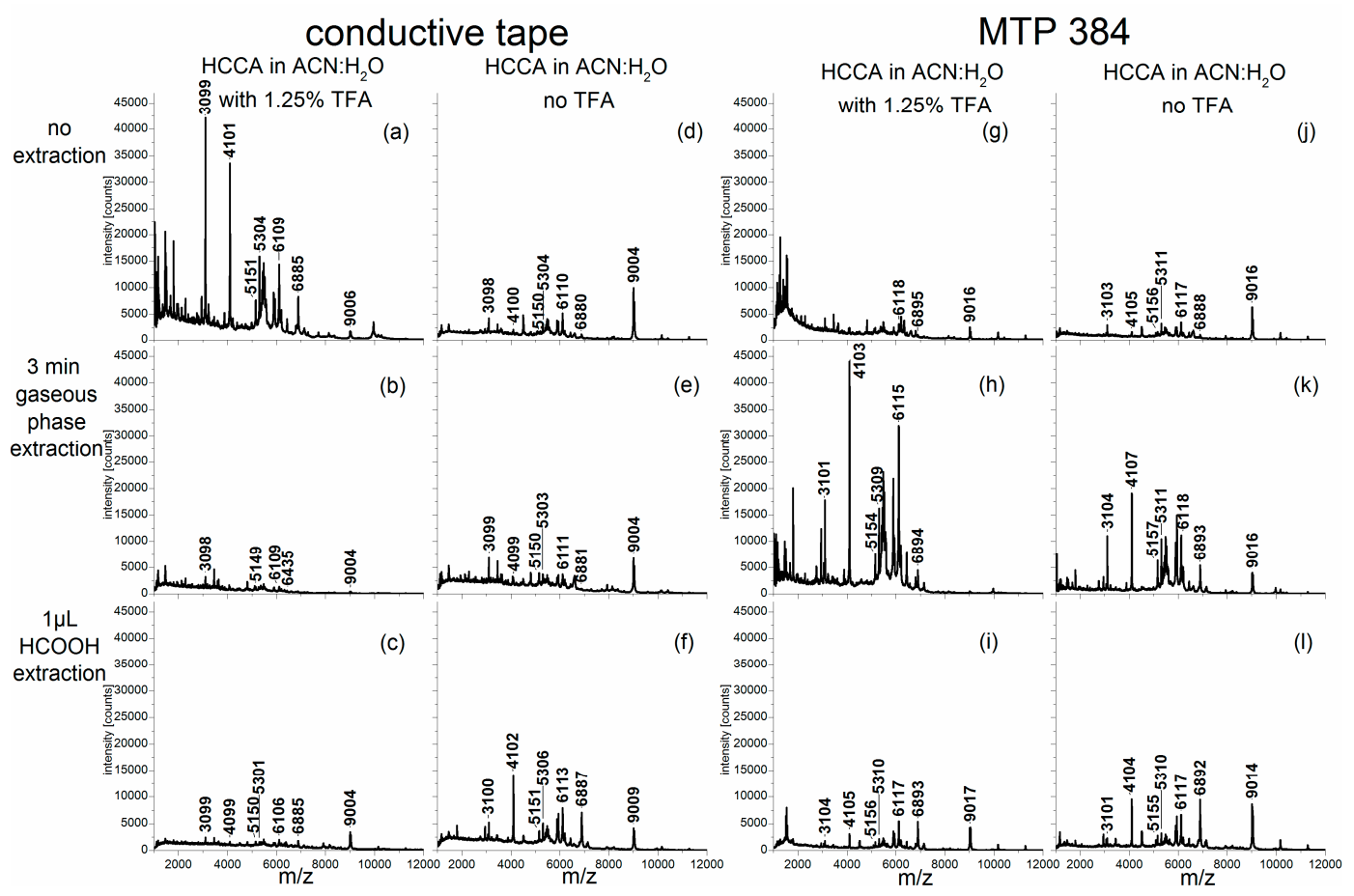

Figure A2. Averaged MALDI-TOF mass spectra $(n=5)$ of Corylus avellana pollen grains: measured on conductive tape with TFA in the matrix solution $(\mathbf{a}-\mathbf{c})$, on conductive tape without TFA in the matrix solution (d-f), on the steel target (MTP 384) with TFA in the matrix solution (g-i) and on the steel target without TFA in the matrix solution ( $\mathbf{j}-\mathbf{1})$ (in the rows: extraction procedure).

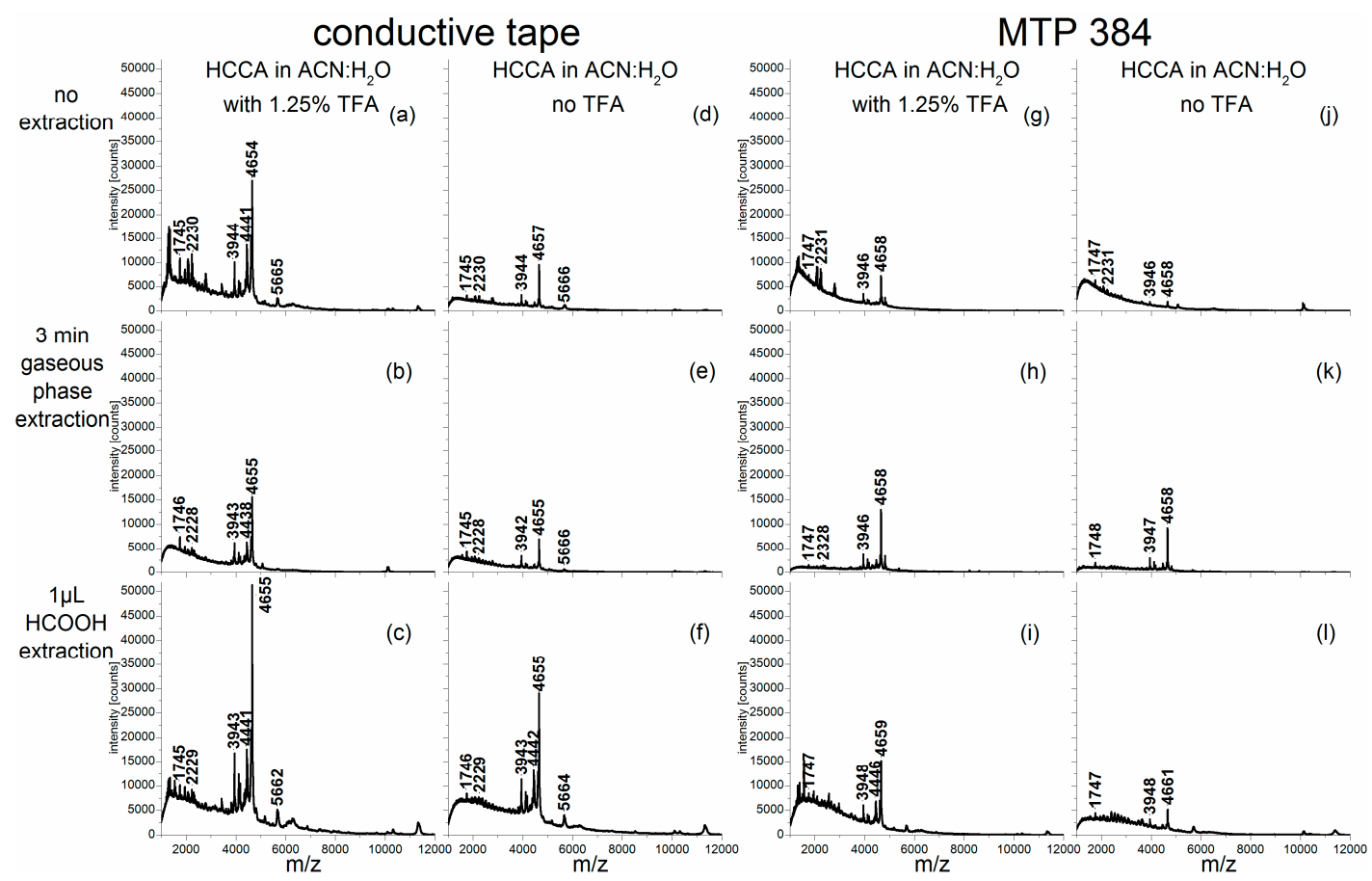

Figure A3. Averaged MALDI-TOF mass spectra $(n=5)$ of Pinus sylvestris pollen grains: measured on conductive tape with TFA in the matrix solution $(\mathbf{a}-\mathbf{c})$, on conductive tape without TFA in the matrix solution (d-f), on the steel target (MTP 384) with TFA in the matrix solution (g-i) and on the steel target without TFA in the matrix solution ( $\mathbf{j}-1)$ (in the rows: extraction procedure). 


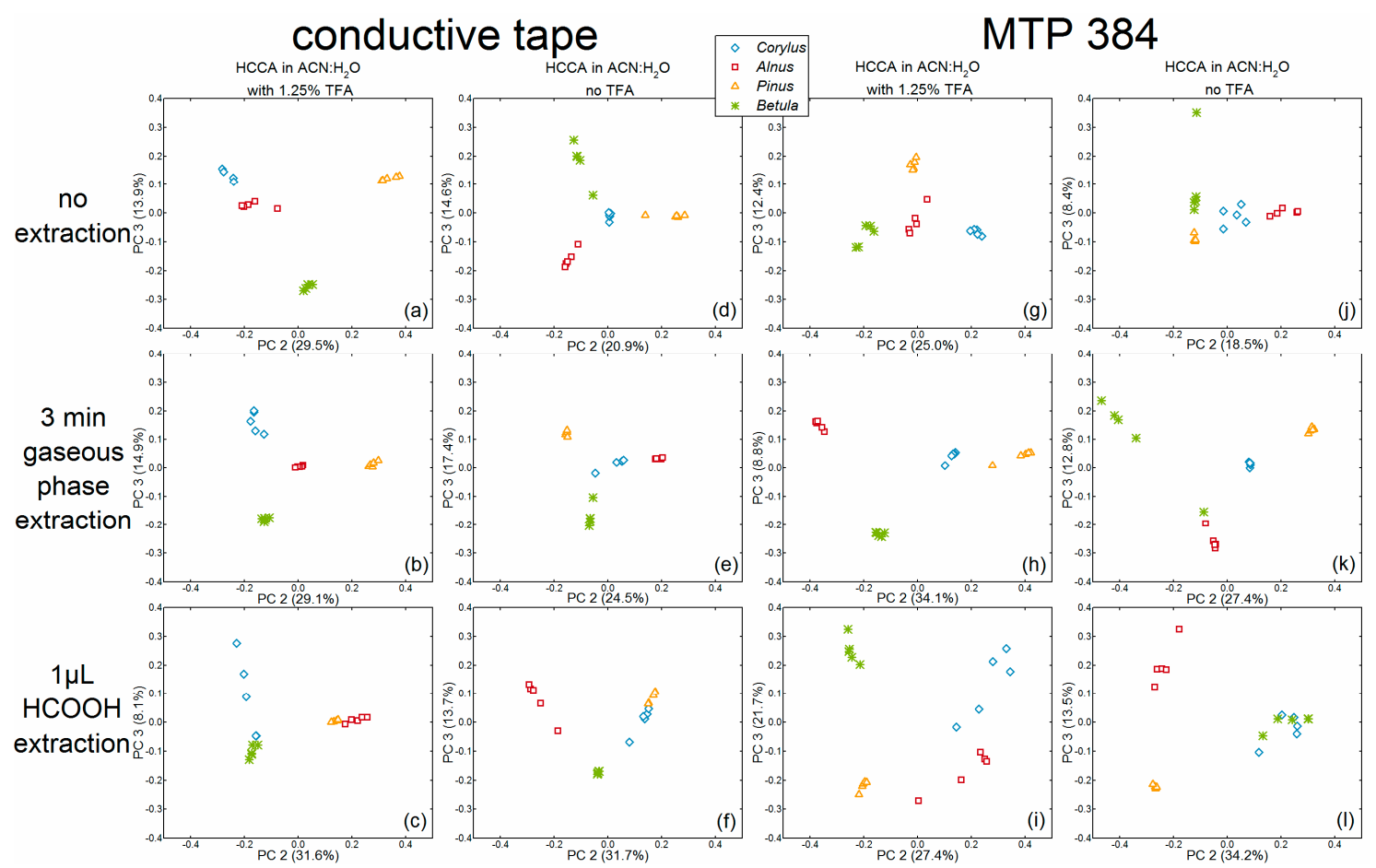

Figure A4. Scores and variances of the second and third principal component (PC) of the PCA of MALDI MS spectra from four pollen species (Corylus avellana, Alnus cordata, Pinus sylvestris, Betula tatewakiana) for different sample preparation techniques (rows: variation in extraction procedure $((\mathbf{a}, \mathbf{d}, \mathbf{g}, \mathbf{j})$ : without additional extraction); (b,e,h, $\mathbf{k})$ : gaseous extraction); (c, $\mathbf{f}, \mathbf{i}, \mathbf{l})$ : droplet extraction); columns: variation in target material and matrix solution $((\mathbf{a}-\mathbf{c})$ : conductive tape with trifluoroacetic acid in the matrix solution); (d-f): CT without TFA in the matrix solution); (g-i): steel target, matrix with TFA); and (j-1): steel target, matrix without TFA)). Data pre-treatment is explained in the methods Section 4.4 .

\section{References}

1. Kaftan, F.; Vrkoslav, V.; Kynast, P.; Kulkarni, P.; Bocker, S.; Cvacka, J.; Knaden, M.; Svatos, A. Mass spectrometry imaging of surface lipids on intact Drosophila melanogaster flies. J. Mass Spectrom. 2014, 49, 223-232. [CrossRef] [PubMed]

2. Tandina, F.; Almeras, L.; Kone, A.K.; Doumbo, O.K.; Raoult, D.; Parola, P. Use of MALDI-TOF MS and culturomics to identify mosquitoes and their midgut microbiota. Parasites Vectors 2016, 9, 495. [CrossRef] [PubMed]

3. Yssouf, A.; Flaudrops, C.; Drali, R.; Kernif, T.; Socolovschi, C.; Berenger, J.M.; Raoult, D.; Parola, P. Matrix-assisted laser desorption ionization-time of flight mass spectrometry for rapid identification of tick vectors. J. Clin. Microbiol. 2013, 51, 522-528. [CrossRef] [PubMed]

4. Lasch, P.; Beyer, W.; Nattermann, H.; Stammler, M.; Siegbrecht, E.; Grunow, R.; Naumann, D. Identification of Bacillus anthracis by using matrix-assisted laser desorption ionization-time of flight mass spectrometry and artificial neural networks. Appl. Environ. Microbiol. 2009, 75, 7229-7242. [CrossRef] [PubMed]

5. Lasch, P.; Drevinek, M.; Nattermann, H.; Grunow, R.; Stämmler, M.; Dieckmann, R.; Schwecke, T.; Naumann, D. Characterization of Yersinia using MALDI-TOF mass spectrometry and chemometrics. Anal. Chem. 2010, 82, 8464-8475. [CrossRef] [PubMed]

6. Lasch, P.; Fleige, C.; Stammler, M.; Layer, F.; Nubel, U.; Witte, W.; Werner, G. Insufficient discriminatory power of MALDI-TOF mass spectrometry for typing of Enterococcus faecium and Staphylococcus aureus isolates. J. Microbiol. Methods 2014, 100, 58-69. [CrossRef] [PubMed] 
7. Krause, B.; Seifert, S.; Panne, U.; Kneipp, J.; Weidner, S.M. Matrix-assisted laser desorption/ionization mass spectrometric investigation of pollen and their classification by multivariate statistics. Rapid Commun. Mass Spectrom. 2012, 26, 1032-1038. [CrossRef] [PubMed]

8. Kajiwara, H.; Hinomoto, N.; Gotoh, T. Mass fingerprint analysis of spider mites (Acari) by matrix-assisted laser desorption/ionization time-of-flight mass spectrometry for rapid discrimination. Rapid Commun. Mass Spectrom. 2016, 30, 1037-1042. [CrossRef] [PubMed]

9. Chaurand, P.; Schwartz, S.A.; Caprioli, R.M. Imaging mass spectrometry: A new tool to investigate the spatial organization of peptides and proteins in mammalian tissue sections. Curr. Opin. Chem. Biol. 2002, 6, 676-681. [CrossRef]

10. Chaurand, P.; Schwartz, S.A.; Reyzer, M.L.; Caprioli, R.A. Imaging mass spectrometry: Principles and potentials. Toxicol. Pathol. 2005, 33, 92-101. [CrossRef] [PubMed]

11. Stoeckli, M.; Chaurand, P.; Hallahan, D.E.; Caprioli, R.M. Imaging mass spectrometry: A new technology for the analysis of protein expression in mammalian tissues. Nat. Med. 2001, 7, 493-496. [CrossRef] [PubMed]

12. Seibert, V.; Wiesner, A.; Buschmann, T.; Meuer, J. Surface-enhanced laser desorption ionization time-of-flight mass spectrometry (SELDI TOF-MS) and ProteinChip ${ }^{\circledR}$ technology in proteomics research. Pathol. Res. Pract. 2004, 200, 83-94. [CrossRef] [PubMed]

13. Muthu, M.; Vimala, A.; Mendoza, O.H.; Gopal, J. Tracing the voyage of SELDI-TOF MS in cancer biomarker discovery and its current depreciation trend-Need for resurrection? Trends Anal. Chem. 2016, 76, 95-101. [CrossRef]

14. Weidner, S.; Schultze, R.D.; Enthaler, B. Matrix-assisted laser desorption/ionization imaging mass spectrometry of pollen grains and their mixtures. Rapid Commun. Mass Spectrom. 2013, 27, 896-903. [CrossRef] [PubMed]

15. Seifert, S.; Weidner, S.M.; Panne, U.; Kneipp, J. Taxonomic relationships of pollens from matrix-assisted laser desorption/ionization time-of-flight mass spectrometry data using multivariate statistics. Rapid Commun. Mass Spectrom. 2015, 29, 1145-1154. [CrossRef] [PubMed]

16. Miki, A.; Katagi, M.; Kamata, T.; Zaitsu, K.; Tatsuno, M.; Nakanishi, T.; Tsuchihashi, H.; Takubo, T.; Suzuki, K. MALDI-TOF and MALDI-FTICR imaging mass spectrometry of methamphetamine incorporated into hair. J. Mass Spectrom. 2011, 46, 411-416. [CrossRef] [PubMed]

17. McDougall, G.J.; Martinussen, I.; Junttila, O.; Verrall, S.; Stewart, D. Assessing the influence of genotype and temperature on polyphenol composition in cloudberry (Rubus chamaemorus L.) using a novel mass spectrometric method. J. Agric. Food Chem. 2011, 59, 10860-10868. [CrossRef] [PubMed]

18. Ng, T.T.; So, P.K.; Zheng, B.; Yao, Z.P. Rapid screening of mixed edible oils and gutter oils by matrix-assisted laser desorption/ionization mass spectrometry. Anal. Chim. Acta 2015, 884, 70-76. [CrossRef] [PubMed]

19. Steinhoff, R.F.; Ivarsson, M.; Habicher, T.; Villiger, T.K.; Boertz, J.; Krismer, J.; Fagerer, S.R.; Soos, M.; Morbidelli, M.; Pabst, M.; et al. High-throughput nucleoside phosphate monitoring in mammalian cell fed-batch cultivation using quantitative matrix-assisted laser desorption/ionization time-of-flight mass spectrometry. Biotechnol. J. 2015, 10, 190-198. [CrossRef] [PubMed]

20. Toh-Boyo, G.M.; Wulff, S.S.; Basile, F. Comparison of sample preparation methods and evaluation of intraand intersample reproducibility in bacteria MALDI MS profiling. Anal. Chem. 2012, 84, 9971-9980. [CrossRef] [PubMed]

21. Hotelling, H. Analysis of a complex of statistical variables into principal components. J. Educ. Psychol. 1933, 24, 417-441. [CrossRef]

22. Bro, R.; Smilde, A.K. Principal component analysis. Anal. Methods 2014, 6, 2812-2831. [CrossRef]

23. Harrington, P.D.B.; Vieira, N.E.; Espinoza, J.; Nien, J.K.; Romero, R.; Yergey, A.L. Analysis of variance-principal component analysis: A soft tool for proteomic discovery. Anal. Chim. Acta 2005, 544, 118-127. [CrossRef]

24. Kessler, W. Multivariate Datenanalyse: Für die Pharma-, Bio- und Prozessanalytik; ein Lehrbuch; Wiley-VCH: Wiemheim, Germany, 2007.

(C) 2017 by the authors. Licensee MDPI, Basel, Switzerland. This article is an open access article distributed under the terms and conditions of the Creative Commons Attribution (CC BY) license (http:/ / creativecommons.org/licenses/by/4.0/). 